\title{
Consciousness Might Be Localized in Extra Physical Dimensions
}

\author{
Ahmad Yousef ${ }^{1}$
}

${ }^{1}$ School of Computational Science and Engineering, McMaster University, Hamilton, Ontario, Canada

*Correspondence: mohamas2@mcmaster.ca

Abstract-In physics, faster travel slows down the time passage until the time stops at the speed of light; afterwards the time starts to have negative values where the past can be "perceptually" accessed. Fasterthan-light particles (tachyons) are assumed to travel in extra physical dimensions, otherwise, the laws of physics will be violated, see Cox, 2012. We think that human consciousness requires tachyons to be formed, physics will be violated, see Cox, 2012 . We think that human consciousness requires tachyons to be form
and this is due to several reasons including but not limited to the necessity of the visual entanglement and this is due to several reasons including but not limited to the necessity of the visual entanglement
between the visual stimulus and the retina to maintain stable visual awareness, see Yousef, 2020. The visual entanglement requires special type of tachyonic dynamics which considers physical processes but in extra physical dimensions. We theorized that the tachyonic regime (faster-than-light) is a sperate world from the baryonic regime (slower-than-light); however, it interacts with our baryonic world through orthogonal physical gates sized way smaller than yoctometers. Based on its nature, the tachyonic regime is dealing with the past, however, it cannot convey any baryonic element to the past. To rephrase, the tachyonic regime can allow the humanity to access "perceptually" the past but not the "physical" past. We believe that human memory requires perfect access to the past; it's therefore the best candidate for comprehending the importance of the playground of the tachyonic dynamics in constructing the human comprehending the importance of the playground of the tachyonic dynamics in constructing the human
consciousness. We also suggest that conscious dreams whether they are due to predictive coding; they are consciousness. We also suggest that conscious dreams whether they are due to predictive coding, they are
subconsciously but fully formulated in the past during and/or just after the perception of the real events. To subconsciously but fully formulated in the past during and/or just after the perception of the real events. To
rephrase, the information of the dreams are formulated in the past and they perceived through nothing but rephrase, the information of the dreams are formulated in the past and they perceived through nothing but
discharges of past memories; discharges established by tachyons to be consciously read by humans. This article will briefly show approaches to this new subject with simplified language and mathematics, however affirmed detection of the tachyons will be exerted in a further conveyance.

The Quantum Entanglement Revisited

We believe that our world consists of two main regimes, the baryonic regime (slower than light), and the tachyonic regime (faster than light). In 1964, John Bell has disproven the Eistein- Podolsky-Rosen paradox in relation of quantum entanglement, and therefore there is spooky action at distane,

in relation of quantum entanglement, and therefore there is spooky action at dist
otherwise tachyons must be existed. The disbelieve of the existence of tachyons

by the scientific community is based on the grandfather paradox, but since our mathematical description for tachyonic dynamics invalidates the possibility of this paradox; there is no reason to disbelieve in tachyons. To rephrase, only tachyons are allowed to travel into these extra physical dimensions; and thus, the grandson will be unable to be physically reconstructed in the past, but to perceive the past. In quantum entanglement, the information is transported between two electrons instantaneously, and this means if there is a tachyon handling the delivery of the information, its speed will be infinity and therefore its energy will approach zero. The technicalities of detecting the tachyons therefore must be very different; and we suggested seven experiments based on our equations to entirely complete the picture suggested in this article.

The Visual Entanglement Reasoned

Scientists reportedly had been reporting that indefinite stable termination of stable binocular

rivalry (BR) is impossible; namely, the frequency of the rivalry must follow the gamma distribution. This is a manifestation of binocular rivalry data (Levelt, 1965; Fox \& Herrmann, 1967; Logothetis, 1998); a signature that is used to discover the neural substrates of binocular rivalry. In our recent reports, however, against the common agreement of the statistical representation of the BR data that includes but not limited the effect of voluntary action on binocular rivalry (see Blake, etal. 2007); we provide several exhibits that reveal indefinite stoppage against binocular rivalry; and thus, the literature of binocular competition should be revisited (see Yousef, 2020). We therefore principlize 'the hidden dynamics' behind these phenomenal terminations, because it is biological impossible to have indefinite local activation of GABA circuits machinery; and thus, the necessity of the extra physical dimensions. The visual entanglement shall ease the understanding of many sensory phenomenon in relation to human attention; these phenomena are including but not limited the invisible gorilla, the attentional blinks, and the perception prior entry hypothesis. We have to emphasize some serious inaccuracies of the IIT "information integration theory of consciousness' advanced by (Giulio Tononi, etal.). This is because of the fact that human subjects can be consciously aware of objects in less than one hundred milliseconds, and this clearly contradicts the neural computations of IIT. Furthermore, human subjects can be visually aware of an object as an intact entity; this means the integration happens before the computational processing! Such fallacies plus further analytical fallacies shall convince the neuroscientific community that novel theories must be offered. We therefore suggest that tachyons are the solution due to its special physical properties in processing the time (manifestation of the information exhibits backward time-shift). Computations within the description of tachyonic dynamics validate the accuracy of such scenarios. But to illustrate further, we have seen how human visual consciousness is conventionally assumed to emerge. But when it comes to face detection, the Fusiform Face Area requires only 50 milliseconds after stimulus onset to exert the corresponding neural activities, see Ghuman, et al. 2014. The causality now appears to be broken; this is because it's computationally impossible for such a process to be achieved in a timely fashion. In another word, it is impossible for the neurons to rightfully compute decisions to deliver, and then afterwards to deliver information related to face detection to the Fusiform Face Area from the retina in 50 milliseconds; the computational impossibility came from many reasons including but not limited to the physiological limitations of the neurons themselves; and therefore ionic entanglements (entangled flags) between the two distant brain areas are suggested; thus an early stage brain area can instantaneously connected to a late one. The aforementioned entangled flags are embedded in our mathematical description for tachyonic dynamics. The terminator neurons, however, are assumed to emit different type of tachyons to impact the consciousness in the presumed extra physical dimensions. Because of the special properties of tachyons in processing the time; the computation of such visual experience is now possible to be achieved timely! Faster-than-Light Reimagined

Energy transformations may cause a system to lose some of its energy content, and thus, according to Einstein's formula, that system will lose some corresponding mass, releasing it as radiant electromagnetic energy, such as light or heat. Now, imagine two identical systems that are continuously losing their mass by radiating photons. With engineering, radiant photons from the first system might achieve complete destructive interferences with radiant photons from the other system. Such interferences cause amounts of the two systems' mass/energy to disappear from our observable universe, \& physicists estimates that these amounts are transported to extra physical dimensions!

But what kind of particles that may be travelling in these extra physical dimensions? According to our thought experiments, they should be faster-than-light particles name of tachyons; whereas the invasion of the light barrier requires subatomic gates (sized way smaller than yoctometers and act as compressor; gates that are way smaller than a million billion of the size of a photon 'assuming that the photon is a

quasiparticle with a radius of $\lambda / \pi^{\prime}$ ). To reiterate, we present simplified theoretical approach of the physical existence of tachyons: Imagine the Laser Interferometer Gravitational-Wave Observatory 'LIGO system' but with extremely short two tubes, namely, the tubes' length has to be the laser's wavelength; the LIGO detector will therefore detect a zero; and this seriously violates the energy conservation principle. To resolve the aforementioned violation; we suggested that the energy were conveyed to extra physical dimensions, but since theoretical physics assumes that these extra dimensions are too small; thus, the momentum of the

particles travelling through these dimensions have to be way smaller than the photons. But we have to be attentive here, because if these particles' momentum have to be way smaller, then their speed
must be way faster; otherwise, the energy conservation principle will be again violated, see the following energy equations for better illustration: $E_{\text {Before destructive interference }}=E_{\text {After destructive interference }} \quad$ (1) $x=\operatorname{det}\left(P_{\text {photon }} \times C\right)=w=\operatorname{det}\left(P_{\text {tachyon }} \times Y\right) \quad(2) ;$

Where the dot product of vectors $x$, and $w \rightarrow 0 ; C$ and $Y$ are the speeds of light, and tachyon respectively, and $P$ is the momentum, but the readers have to be again too careful that these are just mathematical illustrations; but our mathematical description for the tachyonic dynamics will be provided in a further notice. But it's very notable that the scientists had been searching for the "unknown" particles in incorrect manners; to understand the reasons, let's see the Planck's equation: Energy $=$ Planck's constant $\times$ Frequency

But remember that because the LIGO detector shall detect a zero according to our thought experiment; therefore, the resultant particles will have zero value of the frequency; namely, the resultants' wavelength will be infinite. This affirms that cientists had been looking into the wrong planes and dimensions, namely, the Large Hadron Collider 'LHC system' will never be able to directly detect tachyons, unless their

dynamics are considered in the detection design. The existence of tachyons were disbelieved by scientists because there is a major error in understanding this secret world, a misunderstanding that yields the famous grandfather paradox. Our mathematical description for tachyonic dynamics, however, states that such paradox cannot happen because tachyons require to enter through gates much smaller than yoctometers to be emerged 'see the quantum foam for approachable understanding'. Pursuant to our mathematics, it is physically an impossible mission for a human to enter these gates; and therefore, the grandfather paradox is terminated. But scientists will remain skeptical about the existence of tachyon because there is no detective evidence to date. Based on our mathematical description for tachyonic dynamics, tachyons shall have different types: weak tachyons and strong tachyons. Weak tachyons are assumed to be just assistants in completion of quantum processes, and they can be found in the quantum entanglement and quantum tunneling processes; but the detection cannot be established without understanding the extra physical dimensions. In quantum entanglement, the information is transported between two electrons instantaneously, and this means if tachyons handling the delivery of the information, its speed will be infinity and therefore its energy will approach zero. To reiterate, the discovery of the tachyons may not be found through the conventional physical settings; and the strong tachyons has to be discovered before the weak ones to enable the scientists in better detection design, whereas the strongest tachyon (the slowest tachyon) shall have the greatest energy.

Receptors of the Human Cells

Before navigating the literature legacy of the human consciousness; we have first to look at the receptors that generate the qualia. We believe that the human cells consisted of different receptors:

1. Chemoreceptors which can drive several senses including the sense of smell, taste, health, and pain; they can be found in the whole body and medicine relies on them to heal the patients.

Photoreceptors which can drive the sense of vision and the sense of heat; they can be found in the retina (light) and in the skin (infrared photons).

3. Mechanoreceptors which can drive the sense of hearing and touch; they can be found in the inner ear and in the skin. 
4. Tachyceptors which are extremely tiny but undiscovered receptors. They are assumed to drive the sense of the conscious awareness and they're assumed to neighboring the aforementioned three categorized receptors. They are proposed to condensate all of the information inputs into a single wave function to harmonize the conscious experience.

\section{Consciousness: Literature Legacy}

To superficially navigate through the proposed tachyonic dynamics which shall assist in answering the hard problem of consciousness; namely, how and why we have qualia or phenomenal experiences and how sensations acquire characteristics, (Chalmers, 1995); we initially require crediting the previous literature related to human consciousness. There are a few scientists think that consciousness in the universe is scale invariant, (Meijer, 2017); others believes that consciousness is not computational (Penrose, 2014). We do agree with Penrose that consciousness is not computational; access to consciousness however requires computations. Noticeably, the Penrose-Hameroff theory of quantum consciousness claims that microtubules inside the neural cells are structured in a fractal pattern which would enable quantum processes to occur; and therefore, we may say, they implicitly claim that there are computations to access consciousness but alike the conventional ones. Zooming out, Koch, and his collogues had been trying to localize the neural correlate of consciousness (NCC) neurons. But they remain skeptical about whether the frontal lobe is contributing to the creation of consciousness, (Koch et al., 2016a), until we announced the termination of the binocular rivalry (Yousef, 2019). Our concern, however, is to find the precise playground in which vivid human consciousness can be generated.

Consciousness: Searching for the location

We think that any disappearance of the impact of matter/energy (e.g., photons) form our observable universe should refer to extra physical dimensions; dimensions that may include human consciousness. To illustrate our view, let's see how human visual consciousness works. First, photons hit the retinal photoreceptors, that signal the bipolar cells, and those cells synapse the ganglion cells, which signal the brain through a process called, spikes train. Namely, a neuron synapses another neuron which synapses a following neuron, etcetera! The spikes train is ended when it meets an inhibitory (terminator) neuron. Scientific reports had been demonstrating a temporal synchronization between negative activities in Fusiform Face Area "brain region that includes inhibitory neurons and holistically processes faces" and a conscious experience called, face recognition. FFA inhibitory processes are therefore theorized to end up the impact of photons from our detectable universe. Namely, the impact of photons in our measurable universe maybe completely nulled simultaneously with face recognition! That disappearance of matter/energy from our detectable physics has implications to extra dimensions; and we therefore theorize that consciousness maybe placed in extra physical dimensions. Ultimately, our theory concludes the following: the terminator neurons are the neurons which signal the consciousness (the tachyonic playground that has special geometries abstracting the which signal the consciousness (the tachyonic playground that has special geometries abstracting the
information of the universe). Holistically, consciousness should have a spectrum, namely, multiple levels starting from oblivion, or vegetative state, to minimally conscious state, to normal consciousness and ending by the greatest awareness, or metacognition. Greater spikes trains in the cortical regions might assist in creating greater awareness; accessing the consciousness, however, could be only achieved through the inhibitory (terminator) neurons. Since the terminator neurons are distributed in the whole brain, this explains that consciousness is a product of the different parts of the brain; in another word, 'the inner movie that represents the stream of consciousness' (see Chalmers' proposal) is assumed to be result of 'the intermediate neural inhibitions. However, to practically distinguish between two conscious states (face recognition as an example), we assume that early stages cortical areas may directly send face features (skin texture, color, and structure) to the consciousness after the corresponding inhibitory neural processes, but some critical holistic features of the face are not prepared to access the consciousness, thus, they will be sent to the FFA; a region that is assumed to prepare these features to have perfect access to the consciousness. If to the FFA, a region that is assumed to prepare these features to have perfect access to the consciousness. If
neurons in the neurological pathways that link the early stages with the FFA are destroyed, face recognition might be impaired; but not features realization. If those neurological roads remain intact until their signals are delivered to FFA, certain inhibitory patterns will be produced to properly access the consciousness; an thus, face recognition. Luckily, through our several psychophysical trails, we had been able to degrade the human visual consciousness without physical destructions against the neurological pathways to consciousness; namely, by theoretically inhibiting the delivery of sufficient oxygenated hemoglobin to the intended area, see reference 5. Precisely, through manipulations to the attributes of the visual stimulus, we achieved degradation against face recognition "by diminishing the amount of oxygenated hemoglobin in the FFA; aimed to reduce the activities of FFA inhibitory neurons, and therefore, to shorten the neurological pathways to consciousness". This is a straightforward explanation where the time passage is not violated; consciousness is therefore appearing to be baryonic dynamics expect for the last part of the process.

However, there are many circumstances show backward time shift as explained in a previous section. Towards the Validation Process

We believe that the tachyonic playground shall contain guiding signals (weak tachyons) to our conventiona spacetime continuum. For the visual entanglement, we theorize that the inhibitory neurons are tachyonic emitters and the biological receptors are the tachyonic receivers. The termination of the binocular rivalry manifests derivatives from our description for tachyonic dynamics; namely, it exhibits visual entanglement which is different from the electronic (neural) entanglement. To rephrase, the visual entanglement is established when the brain emits two entangled tachyons to create entanglement between the dominating stimulus and the retina (burst of tremendous amount of tachyonic emissions shall be produced to achieve a single visual entanglement). In informal words, each tachyonic emission yielded by attention shall have two 'entangled' tachyons to maintain visual stability. These two entangled tachyons shall arrive to different baryonic position, one to bind with a destinated retinal photoreceptor, and the other is assumed to be tied up with an emitted photon out of an attended visual stimulus; and once the photon is delivered to its entangled photoreceptor, the wave function will be collapsed; and the visual awareness will start its process to emerge.
To biologically validate the visual entanglement theory, however, specialized nano-sensors have to detect different signals' strengths from the retinal ganglion cells of a single eye during visual awareness domination 'high retinal activities' versus suppression 'low retinal activities'; if confirmed, then our theory will have ultimate credibility. Although the setup is complex; however, it will be used to discover secrets behind the conscious dreams, see reference 15 . Since the suggested surgery that shall prove the visual entanglement is extremely expensive; we therefore have to suggest an inexpensive experiment. We reported entanglement is extremely expensive; we therefore have to suggest an inexpensive experiment. We reported
that peripheral viewing - retinal peripheries - are the generators of the illusory motion reversals (IMLs); that peripheral viewing - retinal peripheries - are the generators of the illusory motion reversals (IML
and the reason behind that could be based on anatomical and physiological facts of the peripheries, see reference 20; we however noticed that these IMLs appear through centrally viewing as well, but in a much fewer quantity. To support the main conclusion of our previous study, we however suggested that these IMLs - generated during the central viewing - are due to entanglement between the stimulus and the retinal peripheries. To rationalize our suggestion, we shall understand that Sterzer, etal. have reported distinguished activations in motion sensitive cortical areas such as hMT+/V5 when IMLs alert the human visual awareness; but studying these brain areas cannot be enough to decide the truthfulness of the visual entanglement. Alternatively, by imaging the brain activities — through the fMRI and EEG compatible MRI detectors - of the two IML conditions offered in reference 20 (peripheral viewing versus central viewing); detectors - of the two IML conditions offered in reference 20 (peripheral viewing versus central viewing),
the theory of visual entanglement can be rigorously testified. In another word, if the neurological pathways the theory of visual entanglement can be rigorously testified. In another word, if the neurological pathway
of the IML awareness - starting from the retina to the LGN, passing through the primary visual cortex, of the IML awareness - starting from the retina to the LGN, passing through the primary visual cortex,
and then destinated the hMT+/V5 - show truly high positive correlations for each single brain area when and then destinated the hMT+/V5 - show truly high positive correlations for each single brain are
central versus peripheral viewing conditions are compared; then these correlations may affirm the truthfulness of the visual entanglement.

Needless to say, the aforementioned experiment is considered as a visual motion rivalry experiment; motion induced blindness illusion shall be therefore considered as a visual rivalry experience (alternation of the visual experience without changes in the physical stimulus). But importantly, the implication of 2008 and 2013 MIB publications by Dönner, etal. are therefore assistive to our entanglement theory; and this is because the authors show similar pupil dilations during the MIB illusion trial (consciously removed stimulus) in comparison with a replay trial (physically removed stimulus). This implies that the pupil dilation during MIB does not reflect the endogenous neural processes that initiate the spontaneous perceptual switch in the MIB illusion; and this gives further virtuous reliability towards the theory of visual perceptual switch in the MIB illusion; and this gives further virtuous reliability towards the theory of visual
entanglement. The countless evidence supporting our theory maybe now visiblized, however, the ultimate entanglement. The countless evidence
confirmation requires retinal studies!

Since this article targets the conscious awareness, we suggest retinal studies for the conscious dreams in REM sleep as well as in visual imagery and memory retrieval tasks for a macaque monkey with patched eyes. We suggest surgical operations to implement sensors rings already designed by us to directly sense the optical nerves in the back of the retina; thus, retinal studies without implementation of the sensors within the retina itself. If these sensors detect distinguished signals; then the tachyons must be true, also see reference 15. Despite our ultimate believe in its precision, validating the mathematical description for tachyonic dynamics is however a lengthy journey that requires high standards of research ethics.

Final Statement

In theory, time is bidirectional; it moves forward in the baryonic regime and moves backwards in the tachyonic regime. The validation of the tachyonic dynamics shall stop the trend that assumes the nonlocalit of the human consciousness. If John Bell is still alive; he would reject the nonlocality concept of the human consciousness because his theory has disproven the hidden variable, and the essence of the human

consciousness is constructed through following physical processes. What's more, our mathematics views the quantum entanglement itself to rely on the tachyonic dynamics. The playground of the human consciousnes is therefore residing in extra physical dimensions. Eventually, the concept of the bidirectionality shall reveal the causality in everything in our physical world, therefore, it will rescue the scientific inquiry from its anticipated death!

Transactional References

[1] David Chalmers, (1995). "Facing Up to the Problem of Consciousness,"

[2] Tononi, (2004). "An information integration theory of consciousness." BMC Neuroscience

[3] Meijer (2017). "Consciousness in Universe is Scale Invariant \& Implies Event Horizon of Human Brain."

[4] Christoph Koch, etal. (2016). "Neural correlates of consciousness: progress and problems."

[5] A. Yousef (2019). "Inactivated Peripheral Fusiform Face Area."

[6] Maureen McHugo, etal., 2013. "The emotional attentional blink: what we know so far"

[7] Raymond (1992). "Temporary suppression of visual processing in an RSVP task: An attentional blink?"

[8] A. Yousef (2019). "Quantifying Consciousness: Electrophysiological Perspective."

[6] Fox, \& Herrmann (1967). Stochastic properties of binocular rivalry Alternations.

[10] A. Yousef (2019). "Voluntary Blinks Stop Binocular Rivalry."

[11] A. Yousef (2019). "Motion Triggers Indefinite Stoppage Against Binocular Rivalry."

[12] Yousef, A. (2020). "Voluntary Movements Regulate Binocular Rivalry."

[13] Yousef, A. (2020). "Deep Breathing Governs Binocular Rivalry,"

[14] van Loon et al., (2013). "GABA shapes the dynamics of bistable perception."

[15] A. Yousef (2019). "Tachyons but Not Photons Might Generate Conscious Dreams."

[16] James Hill and Barry Cox (2012). "Einstein's special relativity beyond the speed of light"

[18] Ghuman, etal. (2014). "Dynamic encoding of face information in human fusiform"

[19] Hameroff, and Penrose (2014). "Consciousness in the universe: A review of the 'Orch OR' theory"

[20] Yousef, A. (2019). "Retinal Peripheries Generate Illusory Motion Reversals"

[21] Sterzer, etal. (2002). "Neural Correlates of Spontaneous Direction Reversals in Ambiguous Apparent Visual Motion"

[22] Dönner, (2008) "Opposite neural signatures of motion-induced blindness in human dorsal and ventral visual cortex"

[23] Dönner, (2013) "Retinotopic patterns of correlated fluctuations in visual cortex reflect the dynamics of spontaneous

perceptual suppression."

[24] A. Yousef (2020). "Binocular rivalry terminated." 